\title{
Cosmic Ray Results from the CosmoALEPH Experiment
}

C. Grupen ${ }^{\mathrm{a}}$ N.-O. Hashim ${ }^{\mathrm{ab}}$ B. Jost ${ }^{\mathrm{c}}$ F. Maciuc ${ }^{\mathrm{b}}$ S. Luitz ${ }^{\mathrm{d}}$ A. Mailov ${ }^{\mathrm{a}}$ A.-S. Müller ${ }^{\mathrm{e}}$ A. Putzer ${ }^{\mathrm{f}}$ B. Rensch $^{\mathrm{g}}$ H.-G. Sander ${ }^{\mathrm{h}}$ S. Schmeling ${ }^{\mathrm{c}}$ M. Schmelling ${ }^{\mathrm{b}}$ R. Tcaciuc ${ }^{\mathrm{a}}$ H. Wachsmuth ${ }^{\mathrm{c}}$ Th. Ziegler ${ }^{\mathrm{c}}$ K. Zuber $^{\mathrm{i}}$

${ }^{a}$ Fachbereich Physik, Universität Siegen, D-57068 Siegen, Germany

${ }^{\text {b} M a x-P l a n c k-I n s t i t u t ~ f u ̈ r ~ K e r n p h y s i k, ~ D-69029 ~ H e i d e l b e r g, ~ G e r m a n y ~}$

${ }^{\mathrm{c}} \mathrm{CERN}, \mathrm{CH}-1211$ Geneva, Switzerland

${ }^{\text {d} S L A C ~ M / S ~ 41, ~} 2575$ Sand Hill Rd., Menlo Park, CA 94025, USA

${ }^{\text {e}}$ Forschungszentrum Karlsruhe, Institut für Synchrotronstrahlung, D-76021 Karlsruhe, Germany

${ }^{\mathrm{f}}$ Kirchhoff-Institut für Physik, Universität Heidelberg, D-69120 Heidelberg, Germany

gerphi electronics GmbH, Gewerbering 15, D-83607 Holzkirchen, Germany

${ }^{\mathrm{h}}$ Institut für Physik, Universität Mainz, D-55099 Mainz, Germany

${ }^{\mathrm{i}}$ University of Sussex, Brighton, East Sussex BN1 9QH, England

CosmoALEPH is an experiment operated in conjunction with the ALEPH detector. The ALEPH experiment took data from 1989 until the year 2000 at the Large Electron Positron Collider (LEP) at CERN. It provides, among others, high resolution tracking and calorimetry. CosmoALEPH used this $e^{+} e^{-}$detector for cosmic ray studies. In addition, six scintillator telescopes were installed in the ALEPH pit and the LEP tunnel. The whole experiment operated underground at a vertical depth of 320 meter water equivalent. Data from ALEPH and the scintillator telescopes provide informaton on the lateral distribution of energetic cosmic ray muons in extensive air showers. The decoherence curve of these remnant air shower muons is sensitive to the chemical composition of primary cosmic rays and to the interaction characteristics of energetic hadrons in the atmosphere. An attempt is made to extract the various interdependencies in describing the propagation of primary and secondary cosmic rays through the atmosphere and the rock overburden, and comparing the data with results from Monte Carlo (MC) calculations. Results on the multiplicity distributions of remnant air shower muons in ALEPH are also compared with expectations based on MC simulations. Data on the cosmic ray muon spectrum and charge ratio up to momenta of $3 \mathrm{TeV} / c$ as obtained with the time projection chamber in ALEPH's strong magnetic field are presented. The large number of events also allowed to unambiguously observe muon tridents and to estimate the cross section for this process.

\section{Introduction}

CosmoALEPH integrates the excellent, high precision performance of a general purpose detector for $e^{+} e^{-}$collision experiments with the capabilities of a set of scintillation counter telescopes spread over a distance of $1 \mathrm{~km}$ from the ALEPH pit in the Large Electron Positron Collider (LEP) tunnel. CosmoALEPH measures cos- mic ray muons at $320 \mathrm{~m}$.w.e. underground. The complete array allows to determine the lateral distribution of remnant muons of extensive air showers that develop in the atmosphere. Because of the relatively large energy cutoff $(70 \mathrm{GeV}$ for vertical incidence) CosmoALEPH is sensitive to the early stages of the air shower development. Therefore the decoherence curve of muons should 
be sensitive to the chemical composition of primary cosmic rays.

The excellent spatial and momentum resolution of the time projection chamber in ALEPH immersed in an axial 1.5 Tesla homogeneous magnetic field allows precision measurements of muon spectra and their charge ratio. The high multitrack resolution permits to analyse muon multiplicities of more than 100 particles distributed over an area of $4 \times 4 \mathrm{~m}^{2}$. The exact measurements provide the possibility to

- determine the chemical composition of primary cosmic rays;

- test the muon spectrum against a claim of the quark-gluon plasma hypothesis on the flux of high energy muons [1]; and

- to test the interaction models of different Monte Carlo generators

In addition, the large amount of data also allows to look quantitatively into the muon pair production by muons, a process also of relevance to the MC generation of extensve air showers.

\section{Experimental Setup}

The layout of CosmoALEPH with the relative distances between the different detector stations is shown in figure 1 .

The scintillator stations consist of stacks of plastic scintillators $1-2 \mathrm{~cm}$ thick, $30-40 \mathrm{~cm}$ wide, and $220-250 \mathrm{~cm}$ long, each having photomultipliers at both ends. Two scintillators on top of each other form a stack. Each station of typically $6 \mathrm{~m}^{2}$ consists of 4-8 stacks. An event is recorded if all four phototubes of at least one stack have fired. The ALEPH detector and its performance has been described in detail elsewhere [2]. For the presented analyses only the hadron calorimeter (HCAL, geometrical area $50 \mathrm{~m}^{2}$ ) and the time projection chamber (TPC, geometrical area $16 \mathrm{~m}^{2}$ ) were used. HCAL allows an unambiguous muon identification, and the TPC provides an excellent momentum resolution of $\sigma_{p} / p^{2} \approx$ $2 \cdot 10^{-4} \mathrm{GeV}^{-1}$ allowing a reliable measurement of the muon momentum spectrum up to energies

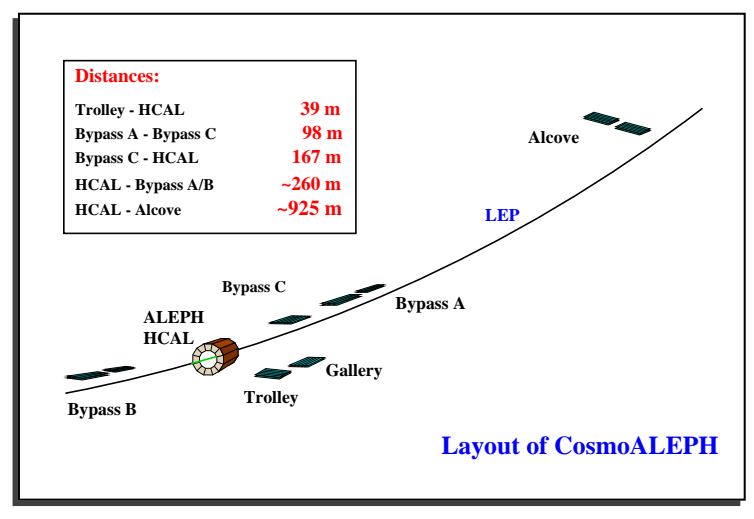

Figure 1. Layout of the CosmoALEPH experiment in the ALEPH pit and the LEP tunnel

of $3 \mathrm{TeV}$. The tracking capability of the TPC enables an angular resolution of $\approx 3$ mrad matching the typical multiple scattering error of muons in the overburden.

The muon momentum cutoff for vertical incidence at $320 \mathrm{~m}$.w.e. is $70 \mathrm{GeV} / c$. Since the overburden is essentially flat in the geometrical acceptance, the effective zenith angle-dependent cutoff is $70 \mathrm{GeV} / \cos \theta$. Most data have been collected using a special HCAL trigger which is active if at least eight planes out of twelve in one HCAL module and eight planes out of twelve in any of the three opposite modules fired simultaneously [3]. In addition, there are also dedicated runs of CosmoALEPH taken with a special cosmic ray trigger without beams in LEP. Analyses have also been made from cosmic ray muons taken in parasitic runs in the presence of LEP beams.

\section{Results}

\subsection{Decoherence Curve}

The lateral distribution of muons in air showers is sensitive to the chemical composition of primary cosmic rays. To infer this chemical abundance requires the knowledge of the spectral shape of the primary energy spectrum and a solid understanding of the interaction characteristics of energetic primaries in the atmosphere. The stan- 
CosmoALEPH (years 1995-2000)
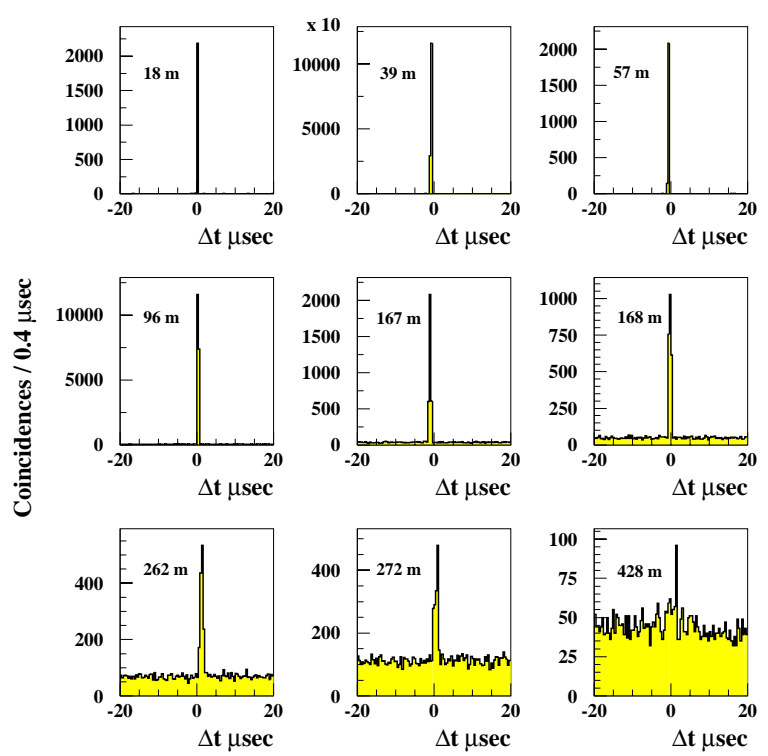

Figure 2. Distribution of arrival time differences for some combinations of detector stations in CosmoALEPH

dard technique to disentangle the various correlations is to use Monte Carlo methods. The MC generators are tuned to accelerator data but the disadvantage is that for the energies in question, accelerator data are not available. Therefore one has to live with extrapolations of cross sections, multiplicities, rapidity distributions and transverse momentum spectra measured from lower energies. An additional problem arises from the fact that accelerator data are mostly taken in different kinematical regimes (low Feynman $x$ ) than required for cosmic ray interactions. Since the different MC generators give somewhat different answers one has to be very careful in the interpretation of the results obtained in this rather indirect way.

Figure 2 shows the distribution of muon arrival time differences for some combinations of detector stations. The signals of muon coincidences are clearly visible and indicate the presence of correlated events from extensive air show-

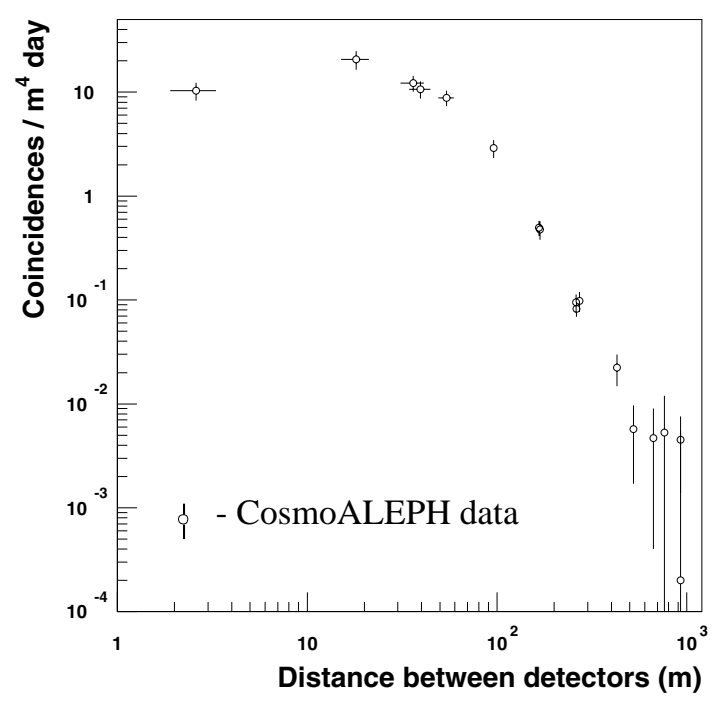

Figure 3. Decoherence distribution of energetic muons underground in CosmoALEPH [4]

ers over distances up to $\sim 500 \mathrm{~m}$. For larger separations the background from chance coincidences starts to become important. The backgroundsubtracted coincidence rates per unit area per unit time have been corrected for geometrical acceptance, different overburden, and detector inefficiencies. From the coincidence rates between various detector stations the lateral distribution of air shower muons underground can be inferred (Figure 3).

This experimentally obtained decoherence curve is shown in figure 4 and 5 in comparison to different MC models [4]. The first point at 2 $\mathrm{m}$ distance was obtained by subdividing the TPC artificially into two halves. The MC curves are the result of a simulation using proton, helium, and iron primaries and two different assumptions on the spectral index of the primary spectrum [4]. The only firm conclusion that one can draw from these lateral distributions is that a light composition is favoured for that part of the primary energy spectrum that is accessible to CosmoALEPH $(\sim \mathrm{PeV}$ region). A detailed interpretation of the lateral distribution requires a better understand- 

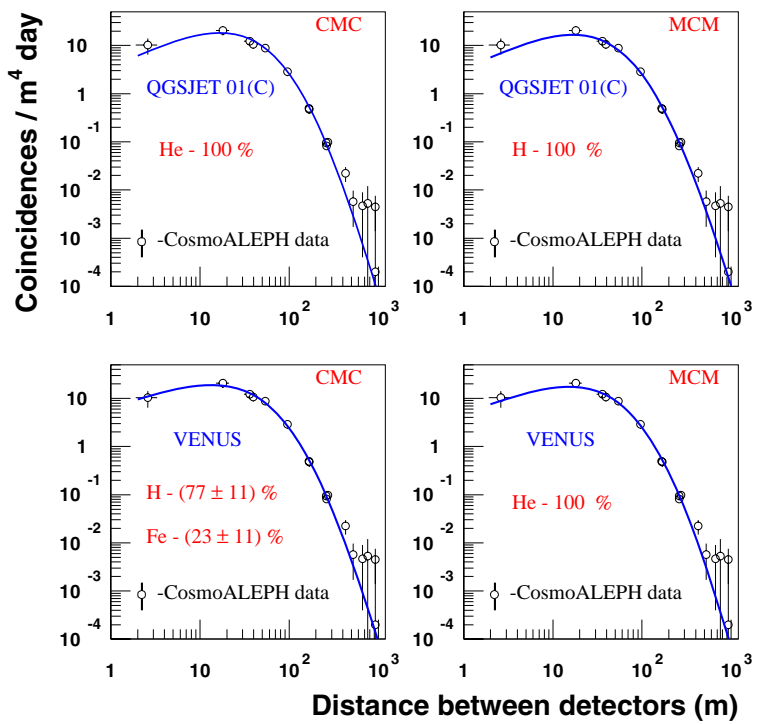

Figure 4. Decoherence distribution of muons underground in CosmoALEPH in comparison to MC models. The MCM version of the primary spectrum has a variable, energy dependent slope for the three elements, while CMC has a common, constant spectral index for all elements. [4]

ing of the interaction characteristics of energetic primaries and their energy spectrum [4].

\subsection{Muon Multiplicity Distribution}

Due to the high spatial resolution of the ALEPH TPC multi-muon events - even with very high multiplicities - can be successfully reconstructed (Figure 6). The muon multiplicity distributions for zenith angles below $30^{\circ}$ and for $30^{\circ} \leq \theta \leq 60^{\circ}$ have been measured separately. Those for the near vertical direction are shown in figure 7 and those for larger zenith angles in figure 8 [5]. For a quantitative analysis the data from ALEPH are compared with expectations from air shower simulations. For this purpose the program CORSIKA (version 5.61) developed by the KASCADE experiment in Karlsruhe was used [6]. The interactions of energetic particles inside CORSIKA were simulated with the QGSJET model which provided an accept-
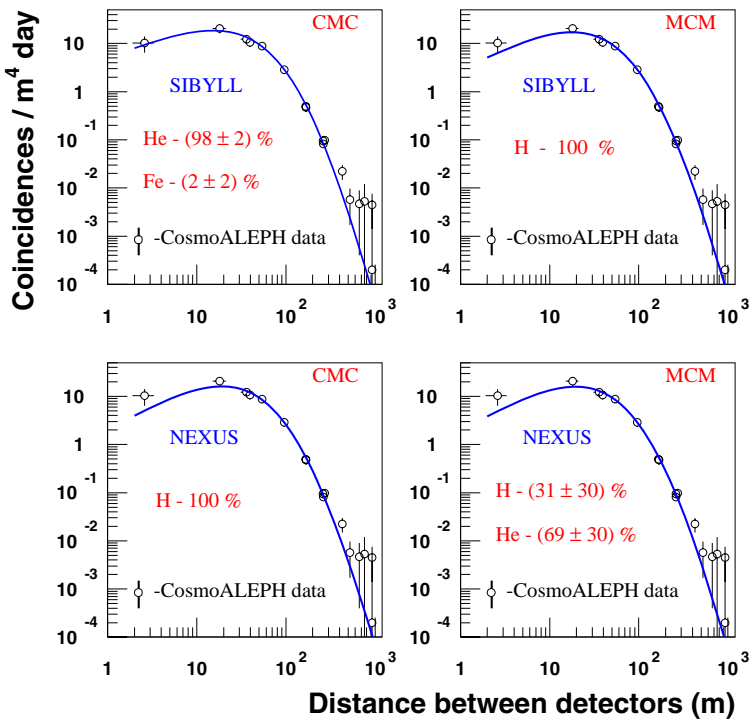

Figure 5. Decoherence distribution of muons underground in CosmoALEPH in comparison to MC models [4]

able description of most of the air shower data. For muon multiplicities $\leq 20$ the distribution can be represented by a proton-dominated primary spectrum for both zenith angular ranges [5]. For higher multiplicities the iron content appears to increase. Iron-induced showers are more effective in producing muons since they interact higher in the atmosphere and also generate a larger number of pions. This appears to be especially true for inclined showers $\left(30^{\circ} \leq \theta \leq 60^{\circ}\right)$. For multiplicities $\geq 70$ event numbers are obtained which appear to be even in disagreement with the assumption of iron primaries. But before claiming that the onset of a quark-gluon plasma [1] or very heavy primaries (uranium ?) are visible in the data, one has to bear in mind that there are only 5 events for multiplicities $\geq 70$ compared to an expectation of just about one for iron. It is, however, worth noting that four of the five high multiplicity events have zenith angles beyond $30^{\circ}$ $[5]$. 


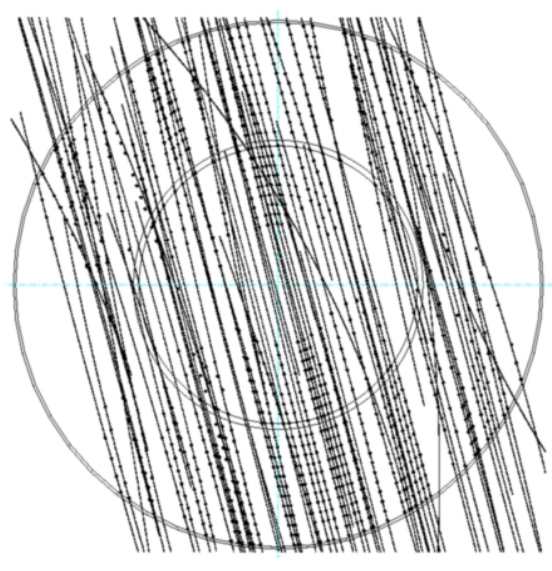

Figure 6. Multi-muon event in the ALEPH TPC demonstrating the high capability to analyse high multitrack events [5]

\subsection{Muon Momentum Spectrum}

For the momentum spectrum measurement only TPC tracks with momenta $p_{\mu} \geq 5 \mathrm{GeV} / c$ were selected. Up to now only muons with near vertical direction of incidence were used. For these muons the acceptance in zenith angle and azimuth is well understood. Also the track reconstruction and trigger efficiency is well under control. The momentum spectrum measured at the ALEPH level underground was corrected for the energy loss in the overburden. Using the known energy-loss relation

$-\frac{\mathrm{d} E}{\mathrm{~d} x}=a+b E$,

where $a$ stands for the ionisation energy loss and $b$ for the energy loss due to direct electron pair production, bremsstrahlung, and nuclear interactions, the surface muon energy $E_{\mu}$ can be calculated from the energy-range relation

$R=\int_{E_{\mu}}^{E_{\aleph}} \frac{\mathrm{d} E}{-\mathrm{d} E / \mathrm{d} x}=\int_{E_{\mu}}^{E_{\aleph}} \frac{\mathrm{d} E}{a+b E}$,

where $E_{\aleph}$ is the energy measured in ALEPH. If $a$ $(=0.21 \mathrm{GeV} / \mathrm{m}$.w.e. $)$ and $b\left(=4 \cdot 10^{-4} \mathrm{~m} \mathrm{w.e.}{ }^{-1}\right)$ are assumed to be constant, one gets

$E_{\mu}=\frac{a}{b} \cdot\left(\mathrm{e}^{b R / \cos \theta}-1\right)+E_{\aleph} \cdot \mathrm{e}^{b R / \cos \theta}$.

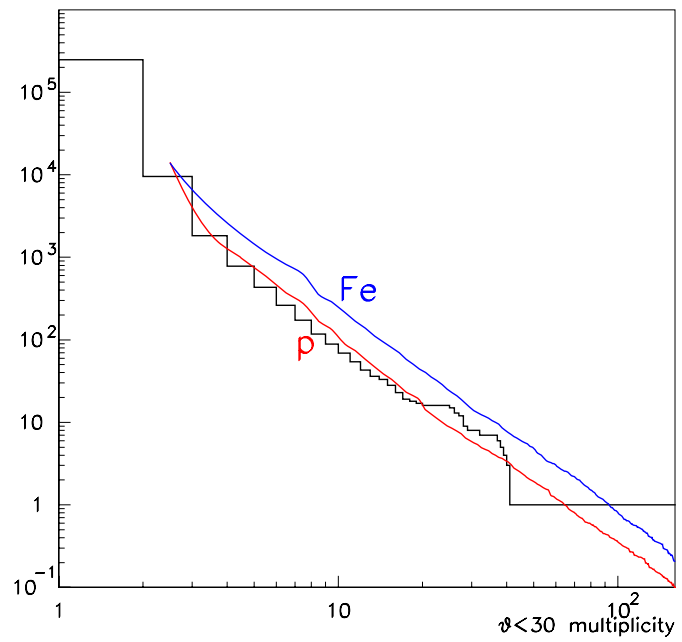

Figure 7. Multiplicity distribution for zenith angles $\leq 30^{\circ}$ in the ALEPH TPC [5]. The data are compared to an absolute prediction based on the QGSJET generator in the CORSIKA program. It is known that QGSJET has shortcomings in the accurate description of the multiplicity distribution. These problems are responsible for the fact that the simulation for protons in the multiplicity range between 4 and 20 shows unphysical values even higher than the data. This small discrepancy gives also an idea on the imperfections of the Monte Carlo simulation.

The sea-level momentum spectrum thus obtained is shown in figure 9 in comparison to other measurements [7]. For convenience the intensity has been scaled by $p^{3}$ as seen in figure 10 . A recent compilation of cosmic ray muon data is also included for comparison [8].

The data are in good agreement with $\mathrm{L} 3+\mathrm{C}$ data [9] and a parametrisation of Bugaev [10]. They agree with a muonic origin from pion and kaon decays.

\subsection{Muon Charge Ratio}

The charge ratio of muons is very robust against systematical errors. It is shown in figure 11 for a zenith angle range of up to $50^{\circ}$. The data show a constant, energy-indepedent ratio of 


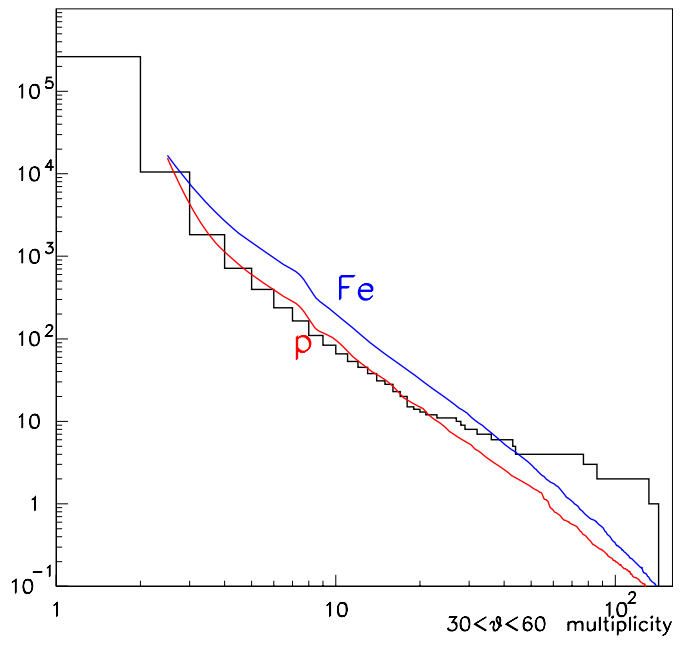

Figure 8. Multiplicity distribution for zenith angles between $30^{\circ}$ and $60^{\circ}$ in the ALEPH TPC [5]

$1.278 \pm 0.011$ averaged over the ALEPH momentum range from $80 \mathrm{GeV} / c$ to $3000 \mathrm{GeV} / c$. The charge ratio also provides a check on the MC generators. Figure 12 shows that the models VENUS 4.12 [11] and DPMJET 2.55 [12] describe the data extremely well, QGSJET 01 [13] is slightly low by about $10 \%$. SIBYLL 2.1 [12] in the present form is clearly ruled out. The prediction of a high value of the charge ratio (around 1.5, slightly increasing with momentum) by SIBYLL is related to a too high multiplicity of $K^{+}$mesons [12]. This is known by now and it is underlined and confirmed by our data. In this aspect, SIBYLL needs to be retuned to take care of the well-measured charge ratio [12].

\subsection{Muon Tridents}

In most cases the differential cross section for muon trident production $\mu+Z \rightarrow \mu+Z+\mu^{+}+\mu^{-}$ is calculated in the framework of QED. The Bugaev-Kotov-Rozental (BKR) formula [14] describes both $\mu^{+} \mu^{-}$and $e^{+} e^{-}$transition rates. This BKR formula was improved by Kokoulin, Kelner, and Petrukhin (KKP) [15] by the inclusion of a form factor for the nucleus. This theoretical cross section treats the nucleon as a quasi-

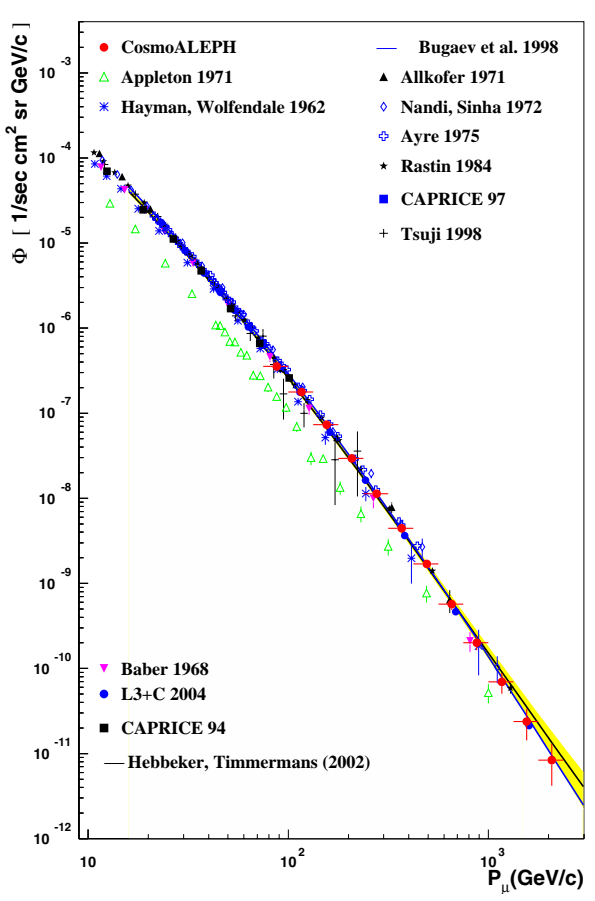

Figure 9. Muon momentum spectrum for near vertical directions as measured in the ALEPH TPC [7]. The references are contained in [8].

Coulomb scatterer with a Fermi function as form factor. A theoretical estimate of the muon trident rate for CosmoALEPH is obtained by propagating muons through the overburden and the detector components of ALEPH.

Figure 12 shows a fully reconstructed trident event in ALEPH [16].

The observed production rate of tridents inside the ALEPH detector (2 events) agrees well with the expectation both in event numbers and particle energies of secondaries based on the KKP parametrisation of the cross section. The BKR estimate neglecting nuclear form factors overestimates the muon trident rate and is strongly disfavoured [16]. Even though this has little effect for the simulation of air showers, the QED parametrisation of this process should in future be corrected by introducing a nuclear form factor. 


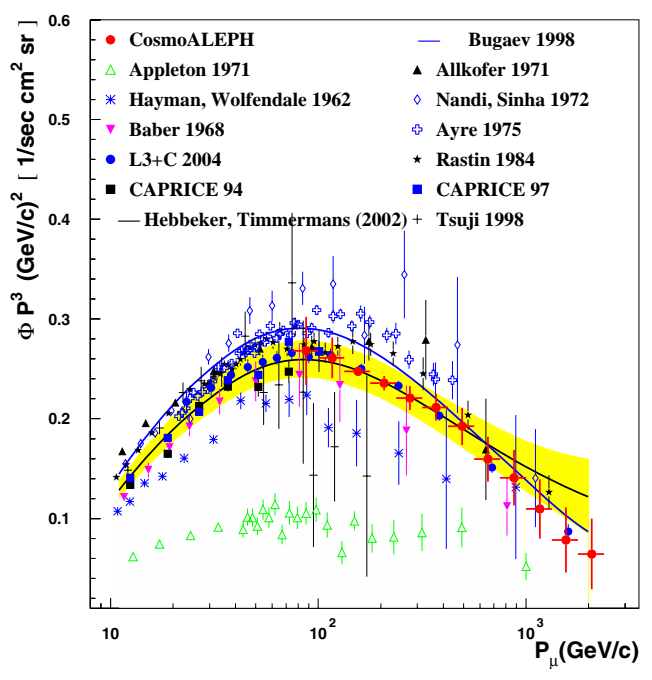

Figure 10. Muon momentum spectrum for near vertical directions scaled with $p^{3}$ as measured in the ALEPH TPC [7]. The references are contained in [8].

\subsection{Conclusions}

The main results from CosmoALEPH can be summarised in the following way:

- The interpretation of the decoherence curve in terms of the chemical composition of primary cosmic rays is strongly influenced by the MC model used and the assumption on the spectral indices of primary particle spectra. More input from accelerator data at higher energies is urgently needed to tune the MC models and to derive firm conclusions about the chemical composition from cosmic ray data. At face value the CosmoALEPH data, which are sensitive to $\mathrm{PeV}$ primary energies, favour a light composition.

- Measurements of the muon multiplicties with the TPC are sensitive to the chemical composition of the primaries. The observed rate of high multiplicty events at large zenith angles is only marginally consistent with current MC models.

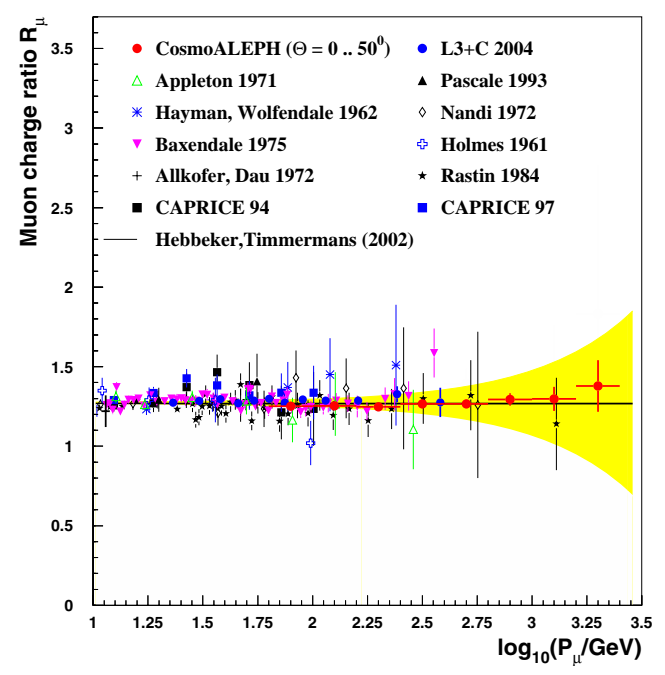

Figure 11. Muon charge ratio for zenith angles up to $50^{\circ}$ as measured in the ALEPH TPC [7]. The references are contained in [8].

- The muon momentum spectrum is in agreement with being the result of standard pion and kaon decays.

- The measured muon charge ratio can help to improve the MC models. The $K^{+}$production rate implemented in SIBYLL needs to be adjusted in order to accommodate the observed charge ratio.

- The theoretical description of the muon trident process requires the introduction of a nuclear form factor. A pure QED calculation overestimates the cross section.

\section{Acknowledgements}

The authors gratefully acknowledge the help of the ALEPH collaboration, in particular the shift crew members of ALEPH, without whose support the data taking would not have been possible. The analyses of the CosmoALEPH experiment have been funded by the Deutsche Forschungsgemeinschaft under Grant DFG/Gr/1796/1-3. The corresponding author (C.G.) expresses his thanks 


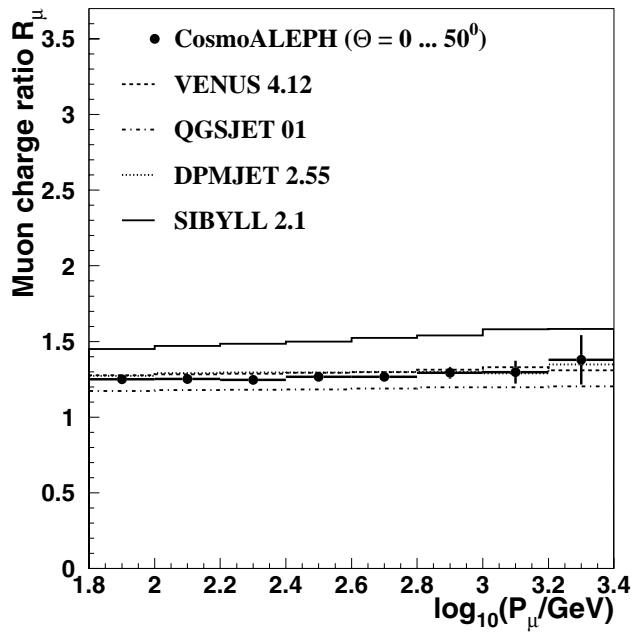

Figure 12. Comparison of the muon charge ratio as measured in the ALEPH TPC compared to various MC models [7].

to the organizers of the XIV International Symposium on Very High Energy Cosmic Ray Interactions, in particular to Prof. Yuqian Ma and Dr. Xinhua Ma for the relaxed atmosphere at Weihai and the hospitality extended to him.

\section{REFERENCES}

1. A. Petrukhin, Manifestations of the QuarkGluon Plasma in Cosmic Ray Experiments; these proceedings, 2006

2. ALEPH Collaboration, Nucl. Instr. Meth. A 360 (1995) 481

3. S. Schmeling, CosmoALEPH Handbook 99001

4. R. Tcaciuc, Ph.D. thesis, University of Siegen, 2006

5. V. Avati et al., Astropart. Phys. 19 (2003) 513

6. J. Knapp, D. Heck, Extensive air shower simulations with the CORSIKA code, Nachr. Forsch. Zentr. Karlsruhe 30 (1998) 27; D. Heck, J. Knapp, J.N. Capdeville, G. Schatz, T. Thouw, CORSIKA: A Monte Carlo Code to Simulate Extensive Air Showers, Report

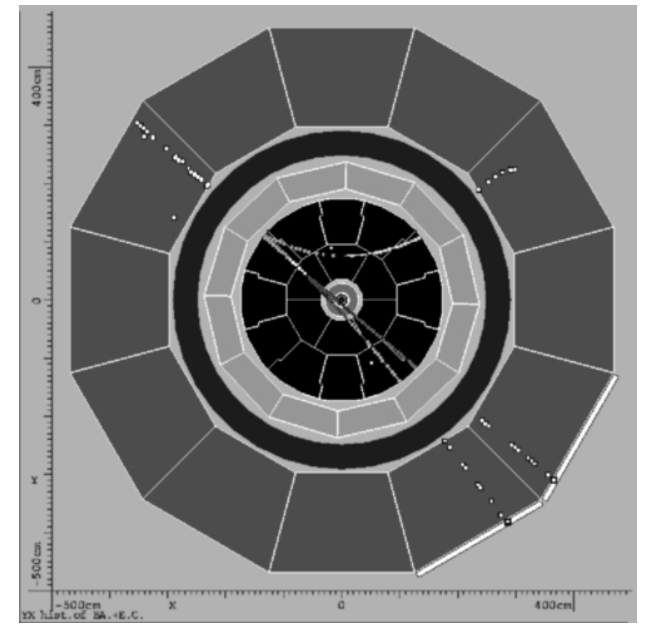

Figure 13. Muon trident event in the ALEPH TPC [16]

FZKA 6019 (1998), Forschungszentrum Karlsruhe

7. N.O. Hashim, Ph.D. thesis, University of Siegen 2006

8. T. Hebbeker and C. Timmermans, Astropart. Phys. 18 (2002) 107

9. P. Le Coultre for the L3+C collaboration, Nucl. Phys. (Proc. Suppl.) 145 (2005) 136

10. E.V. Bugaev et al., Phys. Rev. D 58 (1998) 054001

11. S. Ostapchenko, Nucl. Phys. Proc. Suppl. 52B (1997) 3

12. R. Engel, The Physics of the Models in SIBYLL and DPMJET, these proceedings, 2006

13. S. Ostapchenko, Hadronic Interactions at Cosmic Ray Energies, these proceedings, 2006

14. E.V. Bugaev, Y.D. Kotov and I.L. Rozental, Kosmicheskie myuony i neutrino, Atomizdat, 1970

15. R.P. Kokoulin, S.R. Kelner and A.A. Petrukhin, Proc. Int. Cosmic Ray Conf. Salt Lake City Vol. 2 (1999) 20

16. F. Maciuc et al., Phys. Rev. Lett. 96 (2006) 021801 